\title{
On some functionals of the first passage times in jump models of stochastic volatility
}

\author{
Pavel V. Gapeev* $\quad$ Yavor I. Stoev ${ }^{\dagger}$
}

We compute some functionals related to the generalised joint Laplace transforms of the first times at which two-dimensional jump processes exit half strips. It is assumed that the state space components are driven by Cox processes with both independent and common (positive) exponential jump components. The method of proof is based on the solutions of the equivalent partial integro-differential boundary-value problems for the associated value functions. The results are illustrated on several two-dimensional jump models of stochastic volatility which are based on non-affine analogues of certain mean-reverting or diverting diffusion processes representing closed-form solutions of the appropriate stochastic differential equations.

\section{Introduction}

The main aim of this paper is to derive closed-form expressions for the functionals in (2.9)(2.10) of the first passage times of the two-dimensional jump process $(S, Q)$ defined in (2.1)(2.2). These functionals are related to the associated joint generalised Laplace transforms of the first times at which the continuous-time Markov process $(S, Q)$ exits certain regions forming half strips. It is assumed that the stochastic differential equations in (2.1)-(2.2) for $(S, Q)$ are driven by Cox processes with both independent and common (positive) exponentially distributed jump sizes. Note that the processes of such type can be used for the description of dynamics of the risky asset prices with stochastic volatility rates which play a central role in the modelling of financial assets (see, e.g. Fouque et al. [14], Kallsen [29], and Gatheral [23] for an overview). The consideration of driving Cox processes for the risky asset prices and stochastic volatility rates represents a general feature for the related modern mathematical

${ }^{*}$ London School of Economics, Department of Mathematics, Houghton Street, London WC2A 2AE, United Kingdom; e-mail: p.v.gapeev@lse.ac.uk

$\dagger$ University of Michigan, Department of Mathematics, 530 Church Street, Ann Arbor, MI 48109, United States; e-mail: ystoev@umich.edu

Mathematics Subject Classification 2010: Primary 60J65, 60G40, 60E10. Secondary 34B05, 60J60, 35A09.

Key words and phrases: Two-dimensional jump processes, first exit times, generalised Laplace transforms, stochastic volatility, boundary-value problems, partial integro-differential equations, solvable stochastic differential equations, non-affine processes, mean-reverting and diverting property.

Date: August 12, 2019 
theory. The resulting closed-form expressions for these functionals can be used for calibration of the parameters of the models of financial markets with stochastic volatility. We study the case in which the equation in (2.2) for the mean-reverting or diverting component $Q$ can be either solved explicitly or reduced to the associated ordinary differential equation, by means of an appropriate integrating factor process. Stochastic differential equations of such type were considered by Gard [22; Chapter IV] and Øksendal [37; Chapter V] for diffusion processes, and then in [15] and [20] for their jump analogues. Iyigünler et al. [26] shew the tractability of the resulting analytic solutions of such so-called solvable stochastic differential equations, by means of the analysis of the accuracy of numerical approximations obtained from the appropriate discretisation schemes.

Optimal stopping problems for some mean-reverting and diverting jump processes have been extensively studied in the literature. Davis [8], Peskir and Shiryaev [41]-[42], and Dayanik and Sezer [10]-[11] among others solved optimal stopping problems for such processes arising from the quickest detection of the change points in distributions of the associated discontinuous observable processes (see also [16]-[17]). Mordecki [35]-[36], Kou [30], and Kou and Wang [32] among others solved discounted optimal stopping problems for certain payoff functions depending on the current values of geometric compound Poisson processes with multi-exponential jumps and their various extensions, with the aim of computing rational values for the perpetual American options. Such jump models are widely applied for the description of the dynamics of risky asset prices in financial markets because of their analytical tractability and the property to reproduce the leptokurtic character of the returns distributions. The main feature of these optimal stopping problems and their equivalent free boundary problems was the breakdown of the smooth-fit conditions for the value functions at the stopping boundaries and their replacement by the continuous-fit conditions. Asmussen et al. [3] obtained explicit expressions for the values of the Laplace transforms of the first passage times of more general phase-type Lévy processes over constant boundaries, by means of the Wiener-Hopf factorisation techniques.

Hadjiev [24] studied the problem of computation of the Laplace transforms of the first passage times to upper levels for generalised Ornstein-Uhlenbeck processes with non-positive jumps. Some explicit expressions were obtained for the value functions of the Laplace transforms in a special case of background driving pure jump processes including stable processes. The joint distribution law of the first hitting times of constant boundaries for two constantly correlated drifted Brownian motions was obtained by Iyengar [25]. Analytic expressions for the Laplace transforms of the first passage times of compound Poisson processes over linear boundaries were derived by Zacks et al. [45] in the positive jumps case, and by Perry et al. [38]-[39] in certain cases of positive and negative jumps. Perry et al. [40] and Bo et al. [7] studied the distribution properties of the first rendezvous times of compound Poisson-type processes with Brownian motions and (reflected) Ornstein-Uhlenbeck processes, respectively. Kou and Wang [31] and Sepp [44] obtained closed-form expressions for the Laplace transforms of the first passage times over constant boundaries for double-exponential jump-diffusion processes. Other related stopping problems arising from the computation of the Laplace transforms of the first passage times over constant levels were recently considered by Mijatović and Pistorius [34] for more complicated spectrally positive and negative Lévy processes. Fernandez et al. [13] developed Monte Carlo schemes for the computation of the distribution of the first exit times of jump-diffusion processes from two-sided intervals in the general size distribution case.

The Laplace transforms of the first exit times from connected intervals were recently com- 
puted in [21] for mean-reverting and diverting one-dimensional jump-diffusion processes driven by compound Possion processes with multi-exponential jumps. In the present paper, we derive closed-form expressions for the functionals related to the generalised joint Laplace transforms of the first passage times as stopping problems for a class of two-dimensional jump processes. The latter includes non-affine pure jump analogues of certain mean-reverting and diverting diffusions studied in [20] as the state space components. Aiming to obtain closed-form expressions for the functionals, we assume that the considered jump processes are driven by compound Poisson processes with (positive) exponentially distributed jumps. The same functionals related to the generalised Laplace transforms of the first exit times from half strips were computed in [18] in parallel for a two-dimensional continuous diffusion-type processes with switching coefficients.

It is known that optimal stopping problems for multi-dimensional continuous-time Markov processes are analytically more difficult than the problems for the one-dimensional ones and the solutions of the former are rarely found explicitly. Bayraktar and Poor [6], Bayraktar et al. [5], and Dayanik et al. [9] studied some necessarily multi-dimensional optimal stopping problems arising mostly from the problems of quickest change-point detection for discontinuous Poisson processes and mixed jump-diffusion processes with mean-reverting components. Some analytical results for essentially two-dimensional optimal stopping problems were recently obtained by Gapeev and Shiryaev [19], Johnson and Peskir [28], and Assing et al. [4] in the case of purely continuous diffusion processes. In the present paper, we obtain closed-form solutions to the integro-differential boundary-value problems which are equivalent to the original stopping problems in two-dimensional jump models of stochastic volatility. The exponential distribution of the jumps of the driving compound Poisson processes yields remarkable analytic properties of the value functions of the Laplace transforms at the exit boundaries as well as at the meanreverting and diverting levels in an explicit form. The consideration of the cases in which the mean-reverting or diverting processes contain jumps directed towards the hitting boundaries may lead to the appearance of singularity points in the associated integro-differential operators. These properties can be considered as complements to the breakdown of the smooth-fit principle and its replacement of the principle of continuous fit which was earlier observed in Peskir and Shiryaev [41]-[42] (see also Alili and Kyprianou [2] for necessary and sufficient conditions for the occurrence of smooth-fit condition and references to the related literature).

The paper is organised as follows. In Section 2, we first introduce the setting and notation of the model with a two-dimensional jump process which has the price of a risky asset and the mean-reverting or diverting volatility rate as the state space components. It is assumed that the driving Cox processes have both independent and common single (positive) exponential jump components. We define the functionals related to the generalised joint Laplace transforms of the first exit times from half strips of the two-dimensional jump process and formulate the equivalent boundary-value problem for a partial integro-differential operator. In Section 3, we obtain a closed-form solution to the partial integro-differential boundary-value problem and show that the value function represents the product of solutions of the associated ordinary problems. We derive explicit expressions for the considered functionals in several models based on pure jump analogues of certain continuous diffusion processes solving the appropriate stochastic differential equations. In Section 4, we show that the solutions to the boundary-value problems provide the original functionals of the first exit times. 


\section{Preliminaries}

In this section, we introduce the setting and notation in the problem of computation of some functionals related to the generalised joint Laplace transforms of the first exit times in jump models of stochastic volatility and formulate the associated boundary-value problems.

2.1 The model. Let us consider a probability space $(\Omega, \mathcal{F}, P)$ supporting the processes $X^{j}=\left(X_{t}^{j}\right)_{t \geq 0}, j=1,2,3$, defined by $X_{t}^{j}=\sum_{m=1}^{N_{t}^{j}} Z_{m}^{j}$, where $N^{j}=\left(N_{t}^{j}\right)_{t \geq 0}, j=1,2,3$, which are independent Poisson processes of intensity $\lambda_{j} \geq 0$, and $\left(Z_{m}^{j}\right)_{m \in \mathbb{N}}, j=1,2,3$, are independent exponentially distributed random variables with probability density functions $p_{j}(z)=\alpha_{j} e^{-\alpha_{j} z}$, for all $z>0$ and some $\alpha_{j}>1, j=1,2,3$, fixed. Assume that there exists a process $(S, Q)=\left(S_{t}, Q_{t}\right)_{t \geq 0}$ which provides a (pathwise) unique solution of the system of stochastic differential equations

$$
d S_{t}=S_{t} \delta \sigma^{2}\left(Q_{t}\right) d t+S_{t-} \sum_{j=1}^{2} \int\left(e^{x}-1\right)\left(\mu_{j}-\nu_{j}\right)(d t, d x) \quad\left(S_{0}=s\right)
$$

and

$$
d Q_{t}=\beta\left(Q_{t}\right) d t+Q_{t-} \sum_{j=2}^{3} \int\left(e^{x}-1\right)\left(\mu_{j}-\nu_{j}\right)(d t, d x) \quad\left(Q_{0}=q\right),
$$

for some $s, q>0$ fixed, where $\delta \in \mathbb{R}$ is a constant, and $\beta(q) \in \mathbb{R}$ and $\sigma(q)>0$ are continuously differentiable functions of at most linear growth on $(0, \infty)$ (see, e.g. [33; Chapter IV, Theorem 4.6] and [27; Chapter III, Theorem 2.32] for the existence and uniqueness of solutions of such stochastic differential equations). Here $\mu_{j}(d t, d x)$ is the measure of jumps of the process $Y^{j}=\left(Y_{t}^{j}\right)_{t \geq 0}$ defined by $Y_{t}^{j}=X_{A_{t}}^{j}$, for $j=1,2$, and $Y_{t}^{3}=X_{t}^{3}$, where the process $A=\left(A_{t}\right)_{t \geq 0}$ is given by

$$
A_{t}=\int_{0}^{t} \sigma^{2}\left(Q_{u}\right) d u
$$

for all $t \geq 0$. Then, the compensator of $\mu_{j}(d t, d x)$ with respect to the probability measure $P$ takes the form of $\nu_{j}(d t, d x)=\lambda_{j} \sigma^{2}\left(Q_{t-}\right) \alpha_{j} e^{-\alpha_{j} x} I(x>0) d t d x$, for $j=1,2$, and $\nu_{3}(d t, d x)=$ $\lambda_{3} \alpha_{3} e^{-\alpha_{3} x} I(x>0) d t d x$, respectively (see, e.g. [27; Chapter II, Section 1] for the definitions of these concepts), where $I(\cdot)$ denotes the indicator function. In this case, $Y^{j}, j=1,2$, represent Cox processes of random intensity $\lambda_{j} \sigma^{2}\left(Q_{-}\right)$, while $Y^{3}=X^{3}$ is a compound Poisson process of intensity $\lambda_{3}$ and exponentially distributed with parameter $\alpha_{j}$ jump sizes $Z_{m}^{j}, m \in \mathbb{N}$.

Observe that the process $S$ solving the equation in (2.1) admits the representation

$$
S_{t}=s \exp \left(\int_{0}^{t} \delta \sigma^{2}\left(Q_{u}\right) d u-\sum_{j=1}^{2} \int_{0}^{t} \int\left(e^{x}-1\right) \nu_{j}(d u, d x)+\sum_{j=1}^{2} \int_{0}^{t} \int x \mu_{j}(d u, d x)\right),
$$

for all $t \geq 0$. Note that the process $Q$ forms a one-dimensional (strong) Markov jump-diffusion process, while $(S, Q)$ provides a two-dimensional Markov jump-diffusion process. Without loss of generality and in view of the nature of the problems as well as the examples considered below, we can further assume that the state space of the process $Q$ is $(0, \infty)$, so that the state 
space of the process $(S, Q)$ is $(0, \infty)^{2}$. In this case, the process $S$ can describe the price of a risky asset on a financial market, and $Q$ can represent its volatility rate. Let us finally define the associated with the processes $S$ and $Q$ first passage (stopping) times

$$
\tau_{a}^{-}=\inf \left\{t \geq 0 \mid S_{t} \leq a\right\} \quad \text { and } \quad \tau_{b}^{+}=\inf \left\{t \geq 0 \mid S_{t} \geq b\right\}
$$

as well as

$$
\zeta_{g}^{-}=\inf \left\{t \geq 0 \mid Q_{t} \leq g\right\} \quad \text { and } \quad \zeta_{h}^{+}=\inf \left\{t \geq 0 \mid Q_{t} \geq h\right\},
$$

for some $0<a<b<\infty$ and $0 \leq g<h<\infty$ fixed. The main aim in the present paper is to derive closed form expressions for some functionals related to the generalised joint Laplace transforms of the random times $\tau_{a}^{-}, \tau_{b}^{+}$and $\zeta_{g}^{-}, \zeta_{h}^{+}$.

2.2 Formulation of the problem. In order to formulate the problem of computation of the modified generalised joint Laplace transforms of the random times $\tau_{a}^{-}, \tau_{b}^{+}$and $\zeta_{g}^{-}, \zeta_{h}^{+}$, let us assume that the condition

$$
E\left[\exp \left(\int_{0}^{t \wedge \zeta_{g}^{-} \wedge \zeta_{h}^{+}} \int_{0}^{\infty}\left|e^{\theta_{i} x}-1\right| \lambda_{2} \sigma^{2}\left(Q_{u}\right) \alpha_{2} e^{-\alpha_{2} x} d x d u\right)\right]<\infty
$$

holds, for all $t \geq 0$, where $\theta_{i}, i=1,2$, are some constants which are specified below. In this case, the processes $\left(\widetilde{M}_{t \wedge \zeta_{g}^{-} \wedge \zeta_{h}^{+}}\right)_{t \geq 0}, i=1,2$, with

$$
\widetilde{M}_{t}^{i}=\exp \left(\int_{0}^{t} \int_{0}^{\infty} \theta_{i} x \mu_{2}(d u, d x)-\int_{0}^{t} \int_{0}^{\infty}\left(e^{\theta_{i} x}-1\right) \lambda_{2} \sigma^{2}\left(Q_{u}\right) \alpha_{2} e^{-\alpha_{2} x} d u d x\right)
$$

are uniformly integrable martingales. It thus follows from [43; Appendix, Theorem 6.1] that the probability measures $\widetilde{P}^{i}, i=1,2$, defined by

$$
\left.\frac{d \widetilde{P}^{i}}{d P}\right|_{\mathcal{F}_{t \wedge \zeta_{g}^{-} \wedge \zeta_{h}^{+}}}=\widetilde{M}_{t \wedge \zeta_{g}^{-} \wedge \zeta_{h}^{+}}^{i},
$$

for all $t \geq 0$, are locally equivalent to $P$ on the natural filtration $\left(\mathcal{F}_{t \wedge \zeta_{g}^{-} \wedge \zeta_{h}^{+}}\right)_{t \geq 0}$ generated by the driving processes $Y^{j}, j=1,2,3$.

By applying the change-of-measure arguments, let us now define the functionals $V_{1}^{*}(a ; g, h)$ and $V_{2}^{*}(b ; g, h)$ by

$$
\begin{aligned}
& V_{1}^{*}(a ; g, h)=E\left[e^{-(\eta+\varkappa) A_{\tau_{a}^{-}}} \frac{\widetilde{M}_{\zeta_{h}^{+}}^{1}}{\widetilde{M}_{\tau_{a}^{-}}^{1}} e^{-\varkappa\left(A_{\zeta_{h}^{+}}-A_{\tau_{a}^{-}}\right)} I\left(\tau_{a}^{-}<\zeta_{h}^{+}<\zeta_{g}^{-}\right)\right. \\
&\left.+e^{-(\eta+\varkappa) A_{\zeta_{h}^{+}}} e^{-\eta\left(A_{\tau_{a}^{-}}-A_{\zeta_{h}^{+}}\right)} I\left(\zeta_{h}^{+}<\zeta_{g}^{-} \leq \tau_{a}^{-}<\infty\right)\right] \\
&=E\left[e^{-(\eta+\varkappa) A_{\tau_{a}^{-}}} \widetilde{E}^{1}\left[e^{-\varkappa\left(A_{\zeta_{h}^{+}}-A_{\tau_{a}^{-}}\right)} \mid \mathcal{F}_{\tau_{a}^{-}}\right] I\left(\tau_{a}^{-}<\zeta_{h}^{+}<\zeta_{g}^{-}\right)\right. \\
&\left.+e^{-(\eta+\varkappa) A_{\zeta_{h}^{+}}} E\left[e^{-\eta\left(A_{\tau_{a}^{-}}-A_{\zeta_{h}^{+}}\right)} \mid \mathcal{F}_{\zeta_{h}^{+}}\right] I\left(\zeta_{h}^{+}<\zeta_{g}^{-} \leq \tau_{a}^{-}<\infty\right)\right]
\end{aligned}
$$


and

$$
\begin{aligned}
& V_{2}^{*}(b ; g, h)=E\left[e^{-(\eta+\varkappa) A_{\tau_{b}^{+}}} \frac{\widetilde{M}_{\zeta_{h}^{+}}^{2}}{\widetilde{M}_{\tau_{b}^{+}}^{2}} e^{-\varkappa\left(A_{\zeta_{h}^{+}}-A_{\tau_{b}^{+}}\right)} I\left(\tau_{b}^{+}<\zeta_{h}^{+}<\zeta_{g}^{-}\right)\right. \\
&\left.+e^{-(\eta+\varkappa) A_{\zeta_{h}^{+}}} e^{-\eta\left(A_{\tau_{b}^{+}}-A_{\zeta_{h}^{+}}\right)} I\left(\zeta_{h}^{+}<\zeta_{g}^{-} \leq \tau_{b}^{+}<\infty\right)\right] \\
&=E\left[e^{-(\eta+\varkappa) A_{\tau_{b}^{+}}} \widetilde{E}^{2}\left[e^{-\varkappa\left(A_{\zeta_{h}^{+}}-A_{\tau_{b}^{+}}\right)} \mid \mathcal{F}_{\tau_{b}^{+}}\right] I\left(\tau_{a}^{-}<\zeta_{h}^{+}<\zeta_{g}^{-}\right)\right. \\
&\left.+e^{-(\eta+\varkappa) A_{\zeta_{h}^{+}}} E\left[e^{-\eta\left(A_{\tau_{b}^{+}}-A_{\zeta_{h}^{+}}\right)} \mid \mathcal{F}_{\zeta_{h}^{+}}\right] I\left(\zeta_{h}^{+}<\zeta_{g}^{-} \leq \tau_{b}^{+}<\infty\right)\right],
\end{aligned}
$$

where $\widetilde{E}^{i}$ denotes the expectation taken under the probability measure $\widetilde{P}^{i}$, for $i=1,2$.

In order to compute the values of (2.9)-(2.10), taking into account the strong Markov property, we then introduce the functions $V_{1}^{*}(s, q)=V_{1}^{*}(s, q ; a ; g, h)$ and $V_{2}^{*}(s, q)=V_{2}^{*}(s, q ; b ; g, h)$ defined by

$$
\begin{aligned}
V_{1}^{*}(s, q)=E_{s, q}\left[e^{-(\eta+\varkappa) A_{\tau_{a}^{-}}} U_{1}^{*}\left(Q_{\tau_{a}^{-}}\right) I\left(\tau_{a}^{-}<\zeta_{h}^{+}<\zeta_{g}^{-}\right)\right. \\
\left.+e^{-(\eta+\varkappa) A_{\zeta_{h}^{+}}} W_{1}^{*}\left(S_{\zeta_{h}^{+}}, Q_{\zeta_{h}^{+}}\right) I\left(\zeta_{h}^{+}<\zeta_{g}^{-} \leq \tau_{a}^{-}\right)\right]
\end{aligned}
$$

and

$$
\begin{aligned}
V_{2}^{*}(s, q)=E_{s, q}\left[e^{-(\eta+\varkappa) A_{\tau_{b}^{+}}} U_{2}^{*}\left(Q_{\tau_{b}^{+}}\right) I\left(\tau_{b}^{+}<\zeta_{h}^{+}<\zeta_{g}^{-}\right)\right. & \\
& \left.+e^{-(\eta+\varkappa) A_{\zeta_{h}^{+}}} W_{2}^{*}\left(S_{\zeta_{h}^{+}}, Q_{\zeta_{h}^{+}}\right) I\left(\zeta_{h}^{+}<\zeta_{g}^{-} \leq \tau_{b}^{+}\right)\right],
\end{aligned}
$$

where the functions $W_{1}^{*}(s, q)=W_{1}^{*}(s, q ; a)$ and $W_{2}^{*}(s, q)=W_{2}^{*}(s, q ; b)$, as well as $U_{i}^{*}(q)$, $i=1,2$, are given by

$$
W_{1}^{*}(s, q)=E_{s, q}\left[e^{-\eta A_{\tau_{a}^{-}}} I\left(\tau_{a}^{-}<\infty\right)\right] \text { and } W_{2}^{*}(s, q)=E_{s, q}\left[e^{-\eta A_{\tau_{b}^{+}}} I\left(\tau_{b}^{+}<\infty\right)\right],
$$

as well as

$$
U_{i}^{*}(q)=\widetilde{E}_{q}^{i}\left[e^{-\varkappa A_{\zeta_{h}^{+}}} I\left(\zeta_{h}^{+}<\zeta_{g}^{-}\right)\right]
$$

for $\eta, \varkappa>0$ fixed and all $s, q>0$. Here and after, $\widetilde{E}_{q}^{i}$ and $E_{s, q}$ denote the expectations with respect to the probability measures $\widetilde{P}^{i}, i=1,2$, and $P$ taken under the assumption that the one-dimensional process $Q$ and the two-dimensional process $(S, Q)$ start at $q$ and $(s, q)$ with some $s, q>0$, respectively. We finally note that the stochastic differential equation in (2.2) for the process $Q$ admits the representation

$$
d Q_{t}=\left(\widetilde{\beta}_{i}\left(Q_{t}\right)-\xi_{i} Q_{t}\right) d t+Q_{t-} \sum_{j=2}^{3} \int\left(e^{x}-1\right)\left(\mu_{j}-\widetilde{\nu}_{i, j}\right)(d t, d x) \quad\left(Q_{0}=q\right)
$$

with $\widetilde{\beta}_{i}(q)=\beta(q)+\rho \varepsilon \theta_{i} \sigma(q) \gamma q$, for all $q>0$, as well as

$$
\xi_{i}=\frac{\widetilde{\lambda}_{i, 2}}{\widetilde{\alpha}_{i, 2}-1}-\frac{\lambda_{2}}{\alpha_{2}-1}-\frac{\lambda_{3}}{\alpha_{3}-1}, \quad \widetilde{\lambda}_{i, 2}=\frac{\lambda_{2} \alpha_{2}}{\alpha_{2}-\theta_{i}}, \quad \text { and } \quad \widetilde{\alpha}_{i, 2}=\alpha_{2}-\theta_{i},
$$


whenever $\alpha_{2}>\theta_{i}$ holds, for every $i=1,2$. Here, by means of Girsanov's theorem (see, e.g. [27; Chapter III, Theorem 5.34]), the measure $\widetilde{\nu}_{i, 2}(d t, d x)$ defined by $\widetilde{\nu}_{i, 2}(d t, d x)=e^{\theta_{i} x} \nu_{2}(d t, d x)$ is the compensator of the jump measure $\mu_{2}(d t, d x)$ under $\widetilde{P}^{i}$, and we set $\widetilde{\nu}_{i, 3}(d t, d x)=\nu_{3}(d t, d x)$, for all $t \geq 0$ and every $i=1,2$. It is seen that the process $X^{2}$ represents a compound Poisson process with exponential jumps of intensity $\widetilde{\lambda}_{i, 2}=\lambda_{2} \alpha_{2} /\left(\alpha_{2}-\theta_{i}\right)$ and with parameters of the jump sizes $\widetilde{\alpha}_{i, 2}=\alpha_{2}-\theta_{i}$ under $\widetilde{P}^{i}$, whenever $\alpha_{2}>\theta_{i}$ holds, for $i=1,2$.

2.3 The boundary-value problems. By means of standard arguments based on Itô's formula for semimartingales (see, e.g. [27; Chapter I, Theorem 4.57]), it can be shown that the infinitesimal operator $\mathbb{L}_{(S, Q)}$ of the process $(S, Q)$ from $(2.1)$-(2.2) under the probability measure $P$ acts on a bounded function $V(s, q)$ from the class $C^{2,2}\left((0, \infty)^{2}\right)$ according to the rule

$$
\begin{aligned}
& \left(\mathbb{L}_{(S, Q)} V\right)(s, q)=\left(\delta-\frac{\lambda_{1}}{\alpha_{1}-1}-\frac{\lambda_{2}}{\alpha_{2}-1}\right) \sigma^{2}(q) s \partial_{s} V(s, q) \\
& +\left(\beta(q)-\frac{\lambda_{2} q}{\alpha_{2}-1}-\frac{\lambda_{3} q}{\alpha_{3}-1}\right) \partial_{q} V(s, q)+\int_{0}^{\infty}\left(V\left(s e^{x}, q\right)-V(s, q)\right) \lambda_{1} \sigma^{2}(q) \alpha_{1} e^{-\alpha_{1} x} d x \\
& +\int_{0}^{\infty}\left(V\left(s e^{x}, q e^{x}\right)-V(s, q)\right) \lambda_{2} \sigma^{2}(q) \alpha_{2} e^{-\alpha_{2} x} d x+\int_{0}^{\infty}\left(V\left(s, q e^{x}\right)-V(s, q)\right) \lambda_{3} \alpha_{3} e^{-\alpha_{3} x} d x
\end{aligned}
$$

for all $s, q>0$. The infinitesimal operator $\widetilde{\mathbb{L}}_{Q}^{i}$ of the process $Q$ from (2.15) under the probability measure $\widetilde{P}^{i}$ acts on a function $U(q)$ from the class $C^{2}((0, \infty))$ like

$$
\begin{aligned}
& \left(\widetilde{\mathbb{L}}_{Q}^{i} U\right)(q)=\left(\widetilde{\beta}_{i}(q)-\xi_{i} q\right) U^{\prime}(q) \\
& +\int_{0}^{\infty}\left(U\left(q e^{x}\right)-U(q)\right) \sigma^{2}(q) \widetilde{\lambda}_{i, 2} \widetilde{\alpha}_{i, 2} e^{-\widetilde{\alpha}_{i, 2} x} d x+\int_{0}^{\infty}\left(U\left(q e^{x}\right)-U(q)\right) \widetilde{\lambda}_{i, 3} \widetilde{\alpha}_{i, 3} e^{-\widetilde{\alpha}_{i, 3} x} d x
\end{aligned}
$$

for all $q>0$, where $\widetilde{\beta}_{i}(q)=\beta(q)+\rho \varepsilon \theta_{i} \sigma(q) \gamma q$ and $\xi_{i}$ is given by (2.16), and we set $\widetilde{\lambda}_{i, 3}=\lambda_{3}$ and $\widetilde{\alpha}_{i, 3}=\alpha_{3}$, for $i=1,2$.

In order to find analytic expressions for the unknown value functions from (2.11)-(2.12), let us use the results of general theory of Markov processes (see, e.g. [12; Chapter V]). We reduce the problems of (2.11)-(2.12) for the functions $V_{i}^{*}(s, q), i=1,2$, to the equivalent boundary-value problems for the operator $\mathbb{L}_{(S, Q)}$ of the form

$$
\begin{aligned}
& \left(\mathbb{L}_{(S, Q)} V_{i}-(\eta+\varkappa) \sigma^{2}(q) V_{i}\right)(s, q)=0, \quad \text { for } s>a \text { or } s<b, \\
& V_{i}(s, q)=U_{i}(q), \quad \text { for } s \leq a \text { or } s \geq b \text { and } g \leq q \leq h, \\
& V_{i}(s, q)=W_{i}(s, q), \quad \text { for } q \leq g \text { and } q \geq h \text { and } s \geq a \text { or } s \leq b, \\
& V_{1}(a+, q)=U_{1}(q) \text { or } V_{2}(b-, q)=U_{2}(q), \text { for } g \leq q \leq h, \\
& V_{1}(s, g+)=W_{1}(s, g+) \quad \text { and } V_{2}(s, h-)=W_{2}(s, h-), \text { for } s \geq a \text { or } s \leq b,
\end{aligned}
$$

respectively. Here, the functions $W_{i}(s, q), i=1,2$, solve the problems for the operator $\mathbb{L}_{(S, Q)}$ of the form

$$
\begin{aligned}
& \left(\mathbb{L}_{(S, Q)} W_{i}-\eta \sigma^{2}(q) W_{i}\right)(s, q)=0, \quad \text { for } s>a \text { or } s<b, \\
& W_{1}(s, q)=1, \quad \text { for } \quad s \leq a, \quad \text { and } \quad W_{2}(s, q)=1, \quad \text { for } s \geq b, \\
& W_{1}(a+, q)=1 \text { and } W_{2}(b-, q)=1,
\end{aligned}
$$


for all $q>0$, while the function $U_{i}(q)$ solves the boundary value problem for the operator $\widetilde{\mathbb{L}}_{Q}^{i}$ of the form

$$
\begin{aligned}
& \left(\widetilde{\mathbb{L}}_{Q}^{i} U_{i}-\varkappa \sigma^{2}(q) U_{i}\right)(q)=0, \quad \text { for } g<q<h \\
& U_{i}(q)=0, \quad \text { for } q \leq g, \quad \text { and } U_{i}(q)=1, \quad \text { for } q \geq h, \\
& U_{i}(g+)=0 \text { and } U_{i}(h-)=1
\end{aligned}
$$

for $i=1,2$.

Observe that the appropriate continuity conditions of (2.22)-(2.23), (2.26), and (2.29) can hold only in the cases in which the processes $S$ and $Q$ can pass continuously through the points $a, b$ and $g, h$, respectively. It is remarkable that the stochastic differential equations in (2.1)(2.2) for $S$ and $Q$ do not contain diffusion parts, and thus, the functions $V_{i}^{*}(s, q), W_{i}^{*}(s, q)$, and $U_{i}^{*}(q), i=1,2$, may be discontinuous at the boundaries $a, b$ and $g, h$, depending on the sign of the local drift rates $\left(\delta-\lambda_{1} /\left(\alpha_{1}-1\right)-\lambda_{2} /\left(\alpha_{2}-1\right)\right) \sigma^{2}(q) s$ and $\beta(q)-\xi_{i} q$ in the stochastic differential equations of (2.1)-(2.2) with $\xi_{i}$ of the form of (2.16). The latter property is realised in the case in which the processes $S$ and $Q$ may pass through either of the points only by jumping. Therefore, in order to determine which of the continuity conditions in (2.22)-(2.23), (2.26), and (2.29) should hold for $V_{i}(s, q), W_{i}(s, q)$, and $U_{i}(q), i=1,2$, let us assume that one of the following eight cases is realised. If either the inequality $\delta-\lambda_{1} /\left(\alpha_{1}-1\right)-\lambda_{2} /\left(\alpha_{2}-1\right)<0$ or $\delta-\lambda_{1} /\left(\alpha_{1}-1\right)-\lambda_{2} /\left(\alpha_{2}-1\right)>0$ holds, then the process $S$ can pass through either $a$ or $b$ continuously, and thus, we can assume that $V_{i}(s, q)$ and $W_{i}(s, q), i=1,2$, satisfy either the left-hand or right-hand conditions of (2.22) and (2.26), respectively. If either the inequality $\beta(q)-\xi_{i} q<0$ or $\beta(q)-\xi_{i} q>0$ holds, for all $g \leq q \leq h$, then the process $Q$ can pass through either $g$ or $h$ continuously, and thus, we can assume that $V_{i}(s, q)$ and $U_{i}(q), i=1,2$, satisfy either the left-hand or right-hand conditions of (2.23) and (2.29), respectively. Moreover, if there exists some constant $f$ such that $g<f<h$ and the properties

$$
\beta(q)-\xi_{i} q<0, \quad \text { for } q<f, \quad \beta(q)-\xi_{i} q>0, \quad \text { for } \quad q>f, \quad \text { and } \quad \beta(f)-\xi_{i} f=0
$$

hold, so that the process $Q$ diverts from the level $f$ in a continuous way, and thus, we assume that $V_{i}(s, q)$ and $U_{i}(q), i=1,2$, satisfies both the conditions of (2.23) and (2.29), respectively, since the process $Q$ can pass through $g$ or $h$ continuously. Finally, if there exists some constant $f$ such that $g<f<h$ and the properties

$$
\beta(q)-\xi_{i} q>0, \quad \text { for } \quad q<f, \quad \beta(q)-\xi_{i} q<0, \quad \text { for } \quad q>f, \quad \text { and } \quad \beta(f)-\xi_{i} f=0
$$

hold, then the process $Q$ reverts to the level $f$ in a continuous way, and thus, neither of the conditions of (2.23) and (2.29) is satisfied for $V_{i}(s, q)$ and $U_{i}(q), i=1,2$, respectively, since the process $Q$ cannot pass through $g$ or $h$ continuously.

\section{Solutions to the boundary-value problems}

In this section, we derive closed-form solutions to the boundary-value problems associated with the value functions in (2.11)-(2.14). Note that the analysis presented below provides a significant and detailed addition to the results obtained in [21; Subsection 3.2]. In particular, we observe remarkable analytic properties of the value functions at the boundaries as well as at the mean-reverting and diverting levels in an explicit form. 
3.1 Solutions to the system in (2.27)-(2.29). Let us now assume that $\lambda_{j}>0$ and $\lambda_{5-j}=0$, for some $j=2,3$ fixed. In this case, the integro-differential equation in (2.27) with (2.18) for the unknown function $U_{i, j}(q)$ takes the form

$$
\left(\beta(q)-\xi_{i} q\right) U_{i, 2}^{\prime}(q)-\widetilde{\lambda}_{i, 2} \widetilde{\alpha}_{i, 2} \sigma^{2}(q) q^{\widetilde{\alpha}_{i, 2}} H_{i, 2}(q)-\left(\widetilde{\lambda}_{i, 2}+\varkappa\right) \sigma^{2}(q) U_{i, 2}(q)=0
$$

or

$$
\left(\beta(q)-\xi_{i} q\right) U_{i, 3}^{\prime}(q)-\widetilde{\lambda}_{i, 3} \widetilde{\alpha}_{i, 3} q^{\widetilde{\alpha}_{i, 3}} H_{i, 3}(q)-\left(\widetilde{\lambda}_{i, 3}+\varkappa \sigma^{2}(q)\right) U_{i, 3}(q)=0,
$$

for $g<q<h$, where we set

$$
H_{i, j}(q)=-\int_{q}^{\infty} U_{i, j}(z) z^{-\widetilde{\alpha}_{i, j}-1} d z=-\int_{q}^{h} U_{i, j}(z) z^{-\widetilde{\alpha}_{i, j}-1} d z-\frac{h^{-\widetilde{\alpha}_{i, j}}}{\widetilde{\alpha}_{i, j}},
$$

for all $g \leq q \leq h$, and every $i=1,2$ and $j=2,3$. It follows from the expressions in (3.1)-(3.2) and (2.28)-(2.29) that the function $H_{i, j}(q)$ in (3.3) solves the second-order ordinary differential equation

$$
\begin{aligned}
& \left(\beta(q)-\xi_{i} q\right) q H_{i, 2}^{\prime \prime}(q)-\widetilde{\lambda}_{i, 2} \widetilde{\alpha}_{i, 2} \sigma^{2}(q) H_{i, 2}(q) \\
& +\left(\left(\widetilde{\alpha}_{i, 2}+1\right)\left(\beta(q)-\xi_{i} q\right)-\left(\widetilde{\lambda}_{i, 2}+\varkappa\right) \sigma^{2}(q) q\right) H_{i, 2}^{\prime}(q)=0, \quad \text { for } \quad g<q<h
\end{aligned}
$$

or

$$
\begin{aligned}
& \left(\beta(q)-\xi_{i} q\right) q H_{i, 3}^{\prime \prime}(q)-\widetilde{\lambda}_{i, 3} \widetilde{\alpha}_{i, 3} H_{i, 3}(q) \\
& +\left(\left(\widetilde{\alpha}_{i, 3}+1\right)\left(\beta(q)-\xi_{i} q\right)-\left(\widetilde{\lambda}_{i, 3}+\varkappa \sigma^{2}(q)\right) q\right) H_{i, 3}^{\prime}(q)=0, \quad \text { for } \quad g<q<h,
\end{aligned}
$$

with the boundary conditions

$$
H_{i, j}(h-)=-h^{-\widetilde{\alpha}_{i, j}} / \widetilde{\alpha}_{i, j}, \quad H_{i, j}^{\prime}(g+)=0, \quad \text { and } \quad H_{i, j}^{\prime}(h-)=h^{-\widetilde{\alpha}_{i, j}-1},
$$

for $j=2,3$, where the second and third conditions hold, whenever the inequalities $\beta(q)-\xi_{i} q<0$ and $\beta(q)-\xi_{i} q>0$ are satisfied, for all $g<q<h$, respectively. Observe that the general solutions of the equations in (3.4)-(3.5) are given by

$$
H_{i, j}(q)=C_{i, j, 1} H_{i, j, 1}(q)+C_{i, j, 2} H_{i, j, 2}(q),
$$

where $C_{i, j, k}, i, k=1,2, j=2,3$, are some arbitrary constants, so that the boundary conditions of (3.6) take the form

$$
\begin{aligned}
& C_{i, j, 1} H_{i, j, 1}(h-)+C_{i, j, 2} H_{i, j, 2}(h-)=-h^{-\widetilde{\alpha}_{i, j}} / \widetilde{\alpha}_{i, j}, \\
& C_{i, j, 1} H_{i, j, 1}^{\prime}(g+)+C_{i, j, 2} H_{i, j, 2}^{\prime}(g+)=0, \\
& C_{i, j, 1} H_{i, j, 1}^{\prime}(h-)+C_{i, j, 2} H_{i, j, 2}^{\prime}(h-)=h^{-\widetilde{\alpha}_{i, j}-1},
\end{aligned}
$$

for $i, k=1,2$ and $j=2,3$. Here, the functions $H_{i, j, k}(q), k=1,2$, constitute the fundamental system of solutions of the ordinary differential equations in (3.4)-(3.5), for $g<q<h$, and 
$i=1,2, j=2,3$. Hence, we obtain from (3.3) that the candidate solution for the system in (2.27)-(2.29) admits the representation

$$
U_{i, j}(q ; g, h)=q^{\widetilde{\alpha}_{i, j}+1}\left(C_{i, j, 1}(g, h) H_{i, j, 1}^{\prime}(q)+C_{i, j, 2}(g, h) H_{i, j, 2}^{\prime}(q)\right),
$$

for all $g<q<h$, where the constants $C_{i, j, k}(g, h), i, k=1,2, j=2,3$, are uniquely determined by (3.8) and either the equation in (3.9) or (3.10), whenever either the inequality $\beta(q)-\xi_{i} q<0$ or $\beta(q)-\xi_{i} q>0$ holds, for all $g \leq q \leq h$, respectively. We may therefore conclude that the candidate solution is given by

$$
U_{i, j}(q ; g, h)=-\left(\frac{q}{h}\right)^{\widetilde{\alpha}_{i, j}} \frac{q}{\widetilde{\alpha}_{i, j}} \frac{H_{i, j, 2}^{\prime}(g+) H_{i, j, 1}^{\prime}(q)-H_{i, j, 1}^{\prime}(g+) H_{i, j, 2}^{\prime}(q)}{H_{i, j, 2}^{\prime}(g+) H_{i, j, 1}(h-)-H_{i, j, 1}^{\prime}(g+) H_{i, j, 2}(h-)}
$$

or

$$
\begin{aligned}
U_{i, j}(q ; h)= & \left(\frac{q}{h}\right)^{\widetilde{\alpha}_{i, j}+1} \frac{H_{i, j, 2}(h-) H_{i, j, 1}^{\prime}(q)-H_{i, j, 1}(h-) H_{i, j, 2}^{\prime}(q)}{H_{i, j, 2}(h-) H_{i, j, 1}^{\prime}(h-)-H_{i, j, 1}(h-) H_{i, j, 2}^{\prime}(h-)} \\
& -\left(\frac{q}{h}\right)^{\widetilde{\alpha}_{i, j}} \frac{q}{\widetilde{\alpha}_{i, j}} \frac{H_{i, j, 2}^{\prime}(h-) H_{i, j, 1}^{\prime}(q)-H_{i, j, 1}^{\prime}(h-) H_{i, j, 2}^{\prime}(q)}{H_{i, j, 2}^{\prime}(h-) H_{i, j, 1}(h-)-H_{i, j, 1}^{\prime}(h-) H_{i, j, 2}(h-)},
\end{aligned}
$$

for all $g<q<h$, whenever the inequality $\beta(q)-\xi_{i} q<0$ or $\beta(q)-\xi_{i} q>0$ holds, for all $g \leq q \leq h$ and every $i=1,2$, respectively.

On the other hand, when the condition of (2.30) holds, the candidate solution for the system in (2.27)-(2.29) admits the representation

$$
\begin{aligned}
& U_{i, j}^{+}(q ; f, h)=q^{\widetilde{\alpha}_{i, j}+1}\left(C_{i, j, 1}^{+}(f, h) H_{i, j, 1}^{\prime}(q)+C_{i, j, 2}^{+}(f, h) H_{i, j, 2}^{\prime}(q)\right), \quad \text { for } \quad f<q<h, \\
& U_{i, j}^{-}(q ; g, f)=q^{\widetilde{\alpha}_{i, j}+1}\left(C_{i, j, 1}^{-}(g, f) H_{i, j, 1}^{\prime}(q)+C_{i, j, 2}^{-}(g, f) H_{i, j, 2}^{\prime}(q)\right), \quad \text { for } \quad g<q<f,
\end{aligned}
$$

where the functions $H_{i, j, k}(q), k=1,2$, constitute the fundamental systems of solutions of the ordinary differential equations in (3.4)-(3.5), for $f<q<h$ and $g<q<f$, and every $i=1,2$ and $j=2,3$, respectively. Here, the constants $C_{i, j, k}^{+}(f, h) \equiv C_{i, j, k}^{+}(h), i, k=1,2, j=2,3$, are uniquely determined by the equations in (3.8) and (3.10) with the functions $H_{i, j, k}(q), k=1,2$, while the constants $C_{i, j, k}^{-}(g, f), i, k=1,2, j=2,3$, are uniquely determined by the equation in (3.9) with the functions $H_{i, j, k}(q), i, k=1,2, j=2,3$, as well as the connecting equation

$$
C_{i, j, 1}^{-}(g, f) H_{i, j, 1}(f-)+C_{i, j, 2}^{-}(g, f) H_{i, j, 2}(f-)=C_{i, j, 1}^{+}(h) H_{i, j, 1}(f+)+C_{i, j, 2}^{+}(h) H_{i, j, 2}(f+),
$$

which should hold due to the fact that the distributions of jumps of the process $Y^{j}, j=2,3$, are continuous. We may therefore conclude that $U_{i, j}^{+}(q ; f, h), i, j=1,2$, coincide with $U(q ; h)$ from (3.13), for all $f<q<h$, while $U_{i, j}^{-}(q ; g, f), i, j=1,2$, are given by

$$
U_{i, j}^{-}(q ; g, f)=q^{\widetilde{\alpha}_{i, j}+1} H_{i, j}(f+; h) \frac{H_{i, j, 2}^{\prime}(g+) H_{i, j, 1}^{\prime}(q)-H_{i, j, 1}^{\prime}(g+) H_{i, j, 2}^{\prime}(q)}{H_{i, j, 2}^{\prime}(g+) H_{i, j, 1}(f-)-H_{i, j, 1}^{\prime}(g+) H_{i, j, 2}(f-)},
$$

for all $g<q<f$, with

$$
\begin{aligned}
H_{i, j}(q ; h)= & h^{-\widetilde{\alpha}_{i, j}-1} \frac{H_{i, j, 2}(h-) H_{i, j, 1}(q)-H_{i, j, 1}(h-) H_{i, j, 2}(q)}{H_{i, j, 2}(h-) H_{i, j, 1}^{\prime}(h-)-H_{i, j, 1}(h-) H_{i, j, 2}^{\prime}(h-)} \\
& -\frac{h^{-\widetilde{\alpha}_{i, j}}}{\widetilde{\alpha}_{i, j}} \frac{H_{i, j, 2}^{\prime}(h-) H_{i, j, 1}(q)-H_{i, j, 1}^{\prime}(h-) H_{i, j, 2}(q)}{H_{i, j, 2}^{\prime}(h-) H_{i, j, 1}(h-)-H_{i, j, 1}^{\prime}(h-) H_{i, j, 2}(h-)},
\end{aligned}
$$


for all $f<q<h$, and every $i=1,2$ and $j=2,3$.

Finally, when the condition of (2.31) holds, the candidate solution for the system in (2.27)(2.29) is of the same form of (3.14)-(3.15), where the functions $H_{i, j, 1}^{\prime}(q)$ and $H_{i, j, 2}^{\prime}(q)$ may have singularities on the left- and right-hand side of the point $f$, respectively. In that case, we see that $C_{i, j, 1}^{+}(f, h)=C_{i, j, 2}^{-}(g, f)=0$ should hold, for $i=1,2$ and $j=2,3$, since otherwise $U_{i, j}(q) \rightarrow \pm \infty$ as $q \downarrow f$ and $q \uparrow f$, respectively, that must be excluded, by virtue of the fact that the function $U_{i}^{*}(q)$ in (2.14) is bounded. The remaining constants $C_{i, j, 2}^{+}(f, h) \equiv C_{i, j, 2}^{+}(h)$ and $C_{i, j, 1}^{-}(g, f) \equiv C_{i, j, 1}^{-}(f), i=1,2, j=2,3$, are uniquely determined by the equation in (3.8) and the connecting equation in (3.16). We may therefore conclude that the functions $U_{i, j}^{+}(q ; f, h)=U_{i, j}^{+}(q ; h)$ and $U_{i, j}^{-}(q ; g, f)=U_{i, j}^{-}(q ; f, h), i, j=1,2$, are given by

$$
U_{i, j}^{+}(q ; h)=-\left(\frac{q}{h}\right)^{\widetilde{\alpha}_{i, j}} \frac{q}{\widetilde{\alpha}_{i, j}} \frac{H_{i, j, 2}^{\prime}(q)}{H_{i, j, 2}(h-)}, \quad U_{i, j}^{-}(q ; f, h)=-\left(\frac{q}{h}\right)^{\widetilde{\alpha}_{i, j}} \frac{q}{\widetilde{\alpha}_{i, j}} \frac{H_{i, j, 1}^{\prime}(q)}{H_{i, j, 1}(f-)} \frac{H_{i, j, 2}(f+)}{H_{i, j, 2}(h-)},
$$

for all $f<q<h$ and $g<q<f$, and every $i=1,2$ and $j=2,3$, respectively.

3.2 Some examples. Let us further derive explicit expressions for the fundamental system of solutions $H_{i, j, k}(q), i, k=1,2, j=2,3$, from (3.7), for several volatility and drift rates $\sigma(q)$ and $\beta(q)$ in the stochastic differential equations of (2.1)-(2.2). Other fundamental systems of solutions of the related second-order ordinary differential equations were considered in [21; Examples 3.1-3.3]. It can also be shown that the integrability condition of (2.7) holds in all the cases considered below.

Example 3.1 Let $\sigma(q) \equiv 1$, and $\beta(q)=\left(\beta_{0}-\beta_{1} \ln q\right) q$, for some constants $\beta_{0}, \beta_{1} \in \mathbb{R}$, so that $Q$ is an exponential Ornstein-Uhlenbeck process with jumps, which represents an extended Black-Karasinski model, and thus, the process $(S, \ln Q)$ constitutes a (pure) jump version of the Stein-Stein model of stochastic volatility. Assume, in addition, that $\lambda_{j}>0$ and $\lambda_{5-j}=0$, for some $j=2,3$ fixed. Then, the equations in (3.4)-(3.5) are identical and take the form

$$
\left(\beta_{i, 0}-\beta_{1} \ln q\right) q^{2} H_{i, j}^{\prime \prime}(q)+\left(\left(\widetilde{\alpha}_{i, j}+1\right)\left(\beta_{i, 0}-\beta_{1} \ln q\right) q-\widetilde{\lambda}_{i, j}-\varkappa\right) H_{i, j}^{\prime}(q)-\widetilde{\lambda}_{i, j} \widetilde{\alpha}_{i, j} H_{i, j}(q)=0,
$$

with $\beta_{i, 0}=\beta_{0}-\xi_{i}, i=1,2$, for $g<q<h$. When we assume that $\beta_{1} \neq 0$ holds and the condition in (2.31) is not satisfied, by performing the change of variable $F(q)=\ln q$, it follows from [46; Formulas 2.1.2.108 and 2.1.2.70] that the fundamental solutions $H_{i, j, k}(q), i, k=1,2$, from (3.7) of the equations in (3.4)-(3.5) take the form

$$
H_{i, j, 1}(q)=q^{v_{i, j}} \Psi\left(\varphi_{i, j}, \psi_{i, j} ; R_{i, j}(q)\right) \quad \text { and } \quad H_{i, j, 2}(q)=q^{v_{i, j}} \Phi\left(\varphi_{i, j}, \psi_{i, j} ; R_{i, j}(q)\right)
$$

for $\psi_{i, j} \neq 0,-1,-2, \ldots$, and

$$
\begin{aligned}
& H_{i, j, 1}(q)=q^{v_{i, j}} R_{i, j}(q)^{1-\psi_{i, j}} \Psi\left(\varphi_{i, j}-\psi_{i, j}+1,2-\psi_{i, j} ; R_{i, j}(q)\right), \\
& H_{i, j, 2}(q)=q^{v_{i, j}} R_{i, j}(q)^{1-\psi_{i, j}} \Phi\left(\varphi_{i, j}-\psi_{i, j}+1,2-\psi_{i, j} ; R_{i, j}(q)\right),
\end{aligned}
$$

for $\psi_{i, j}=0,-1,-2, \ldots$, and $i, k=1,2, j=2,3$. Here, we set $v_{i, j}=-\widetilde{\alpha}_{i, j} I_{\left\{\beta_{1}>0\right\}}$, $\varphi_{i, j}=\left(\widetilde{\lambda}_{i, j} I_{\left\{\beta_{1}<0\right\}}+\varkappa I_{\left\{\beta_{1}>0\right\}}\right) / \beta_{1}, \psi_{i, j}=\left(\widetilde{\lambda}_{i, j}+\varkappa\right) / \beta_{1}$, and $R_{i, j}(q)=\operatorname{sgn}\left(\beta_{1}\right) \widetilde{\alpha}_{i, j}\left(\ln q-\beta_{i, 0} / \beta_{1}\right)$, 
and the functions $\Phi(x, y ; z)$ and $\Psi(x, y ; z)$ are are the Kummer's and Tricomi's confluent hypergeometric functions defined by

$$
\Phi(x, y ; z)=1+\sum_{k=1}^{\infty} \frac{(x)_{k}}{(y)_{k}} \frac{z^{k}}{k !}
$$

and

$$
\Psi(x, y ; z)=\frac{\Gamma(1-y)}{\Gamma(x+1-y)} \Phi(x, y ; z)+\frac{\Gamma(y-1)}{\Gamma(x)} z^{1-y} \Phi(x+1-y, 2-y ; z),
$$

for $y \neq 0,-1,-2, \ldots$, where $(u)_{k}$ is the Pochhammer symbol defined as $(u)_{k}=u(u+1) \cdots(u+$ $k-1)$, and $(u)_{0}=1$, for $u \in \mathbb{R}$ and $k \in \mathbb{N}, \Gamma(z)$ denotes the Euler's gamma function, and the series in (3.24) converges under all $z>0$ (see, e.g. [1; Chapter XIII]). Note that the functions in (3.24) and (3.25) admit the integral representations

$$
\Phi(x, y ; z)=\frac{\Gamma(y)}{\Gamma(x) \Gamma(y-x)} \int_{0}^{1} e^{z v} v^{x-1}(1-v)^{y-x-1} d v
$$

for $y>x>0$, and all $z \in \mathbb{R}$, and

$$
\Psi(x, y ; z)=\frac{1}{\Gamma(y)} \int_{0}^{\infty} e^{-z v} v^{x-1}(1+v)^{y-x-1} d v
$$

for $x>0$ and all $z>0$ (see, e.g. [1; Chapter XIII]), respectively.

When the condition in (2.31) is satisfied, we have that $g<e^{\beta_{i, 0} / \beta_{1}}<h, \beta_{1}>0$, and the equation in (3.20) has a singular point at $q=e^{\beta_{i, 0} / \beta_{1}}$, for $g<q<h$. Hence, the properties that the equalities $\Phi(x, y ; 0)=1, \partial_{z} \Phi(x, y ; z)=(x / y) \Phi(x+1, y+1 ; z), \partial_{z} \Psi(x, y ; z)=-x \Psi(x+1, y+$ $1 ; z)$, and $\Psi(x, y ; 0+)=\infty$, for $y>1$, are satisfied yield the fact that $\left|H_{i, j, 2}^{\prime}\left(e^{\beta_{i, 0} / \beta_{1}} \pm\right)\right|=\infty$ holds and the candidate solution for the system in (2.27)-(2.29) is of the form (3.19).

On the other hand, when we assume that $\beta_{i, 0} \neq 0$ and $\beta_{1}=0$ holds, the equation (3.20) takes the form

$$
\beta_{i, 0} q^{2} H_{i, j}^{\prime \prime}(q)+\left(\beta_{i, 0}\left(\widetilde{\alpha}_{i, j}+1\right)-\widetilde{\lambda}_{i, j}-\varkappa\right) q H_{i, j}^{\prime}(q)-\widetilde{\lambda}_{i, j} \widetilde{\alpha}_{i, j} H_{i, j}(q)=0,
$$

for $g<q<h$, and none of the conditions (2.30)-(2.31) is satisfied. We can therefore conclude from [46; Formula 2.1.123] that the candidate solution for the system in (2.27)-(2.29) is of the form (3.12)-(3.13), where we have $H_{i, j, k}(q)=q^{v_{i, j, k}}$ with

$$
v_{i, j, k}=\frac{\widetilde{\lambda}_{i, j}+\varkappa}{2 \beta_{i, 0}}-\frac{\widetilde{\alpha}_{i, j}+1}{2}+(-1)^{k} \sqrt{\left(\frac{\widetilde{\lambda}_{i, j}+\varkappa}{2 \beta_{i, 0}}-\frac{\widetilde{\alpha}_{i, j}+1}{2}\right)^{2}+\frac{\widetilde{\lambda}_{i, j} \widetilde{\alpha}_{i, j}}{\beta_{i, 0}}}
$$

for $i, k=1,2, j=2,3$.

Example 3.2 Let $\sigma(q)=\sqrt{q}$, and $\beta(q)=-\beta_{1} q$, for some constant $\beta_{1} \neq 0$, so that $\ln ^{2} Q / 4$ is a (pure) jump analogue of the Feller square root process which represents an extended CoxIngersoll-Ross model, and thus, the process $\left(S, \ln ^{2} Q / 4\right)$ constitutes a (pure) jump version of the Heston model of stochastic volatility. Assume, in addition, that $\lambda_{j}>0$ and $\lambda_{5-j}=0$, for 
some $j=2,3$ fixed. We first note that in this case none of the conditions of $(2.30)-(2.31)$ is satisfied. Then, the equations in (3.4)-(3.5) take the form

$$
\beta_{i, 1} q H_{i, 2}^{\prime \prime}(q)+\left(\left(\widetilde{\alpha}_{i, 2}+1\right) \beta_{i, 1}+\left(\widetilde{\lambda}_{i, 2}+\varkappa\right) q\right) H_{i, 2}^{\prime}(q)+\widetilde{\lambda}_{i, 2} \widetilde{\alpha}_{i, 2} H_{i, 2}(q)=0
$$

and

$$
\beta_{i, 1} q^{2} H_{i, 3}^{\prime \prime}(q)+\left(\left(\widetilde{\alpha}_{i, 3}+1\right) \beta_{i, 1} q+\varkappa q^{2}+\lambda_{i} q\right) H_{i, 3}^{\prime}(q)+\widetilde{\lambda}_{i, 3} \widetilde{\alpha}_{i, 3} H_{i, 3}(q)=0,
$$

for $g<q<h$, respectively, with $\beta_{i, 1}=\beta_{1}+\xi_{i}$, for $i, k=1,2, j=2,3$. It follows from [46; Formulas 2.1.2.108 and 2.1.2.70] that the fundamental solutions $H_{i, 2, k}(q), i, k=1,2$, from (3.7) of the equation in (3.30) take the form

$$
H_{i, 2,1}(q)=\Psi\left(\varphi_{i, 2}, \psi_{i, 2} ; R_{i, 2}(q)\right) \quad \text { and } \quad H_{i, 2,2}(q)=\Phi\left(\varphi_{i, 2}, \psi_{i, 2} ; R_{i, 2}(q)\right),
$$

for $\psi_{i, 2} \neq 0,-1,-2, \ldots$, and

$$
\begin{aligned}
& H_{i, 2,1}(q)=R_{i, 2}(q)^{1-\psi_{i, 2}} \Psi\left(\varphi_{i, 2}-\psi_{i, 2}+1,2-\psi_{i, 2} ; R_{i, 2}(q)\right), \\
& H_{i, 2,2}(q)=R_{i, 2}(q)^{1-\psi_{i, 2}} \Phi\left(\varphi_{i, 2}-\psi_{i, 2}+1,2-\psi_{i, 2} ; R_{i, 2}(q)\right),
\end{aligned}
$$

for $\psi_{i, 2}=0,-1,-2, \ldots$ Here, we set $\varphi_{i, 2}=\widetilde{\lambda}_{i, 2} \widetilde{\alpha}_{i, 2} /\left(\widetilde{\lambda}_{i, 2}+\varkappa\right), \psi_{i, 2}=\widetilde{\alpha}_{i, 2}+1$, and $R_{i, 2}(q)=$ $-\left(\widetilde{\lambda}_{i, 2}+\varkappa\right) q / \beta_{i, 1}$, for $i=1,2$, and the functions $\Phi(x, y ; z)$ and $\Psi(x, y ; z)$ are defined in (3.26)$(3.27)$.

On the other hand, it follows from [46; Formulas 2.1.139 and 2.1.108] that the fundamental solutions $H_{i, 3, k}(q), i, k=1,2$, from (3.7) of the equation in (3.31) take the form

$$
\begin{aligned}
& H_{i, 3,1}(q)=q^{v_{i, 3}} e^{-\varkappa q /\left(2 \beta_{i, 1}\right)} \Psi\left(\pi_{i, 3} / 2-v_{i, 3}, \pi_{i, 3} ; R_{i, 3}(q)\right), \\
& H_{i, 3,2}(q)=q^{v_{i, 3}} e^{-\varkappa q /\left(2 \beta_{i, 1}\right)} \Phi\left(\pi_{i, 3} / 2-v_{i, 3}, \pi_{i, 3} ; R_{i, 3}(q)\right)
\end{aligned}
$$

for $\pi_{i, 3} \neq 0,-1,-2, \ldots$, and

$$
\begin{aligned}
& H_{i, 3,1}(q)=q^{v_{i, 3}} e^{-\varkappa q /\left(2 \beta_{i, 1}\right)} R_{i, 3}(q)^{1-\pi_{i, 3}} \Psi\left(1-\pi_{i, 3} / 2-v_{i, 3}, 2-\pi_{i, 3} ; R_{i, 3}(q)\right), \\
& H_{i, 3,2}(q)=q^{v_{i, 3}} e^{-\varkappa q /\left(2 \beta_{i, 1}\right)} R_{i, 3}(q)^{1-\pi_{i, 3}} \Phi\left(1-\pi_{i, 3} / 2-v_{i, 3}, 2-\pi_{i, 3} ; R_{i, 3}(q)\right),
\end{aligned}
$$

for $\pi_{i, 3}=0,-1,-2, \ldots$, and $i=1,2$. Here, we set $\pi_{i, 3}=2 v_{i, 3}+\widetilde{\alpha}_{i, 3}+1+\widetilde{\lambda}_{i, 3} / \beta_{i, 1}, R_{i, 3}(q)=$ $\varkappa q / \beta_{i, 1}$, and either $v_{i, 3}=-\widetilde{\lambda}_{i, 3} / \beta_{i, 1}$ or $v_{i, 3}=-\widetilde{\alpha}_{i, 3}$, for $i=1,2$.

3.3 Solutions to the system in (2.24)-(2.26). Let us assume that $\lambda_{j}>0$ and $\lambda_{3-j}=0$, for some $j=1,2$. In this case, the integro-differential equations for the unknown functions $W_{i}(s, q)$ in (2.24) with (2.17) take the form

$$
\begin{aligned}
& \left(\delta-\frac{\lambda_{j}}{\alpha_{j}-1}\right) \sigma^{2}(q) s \partial_{s} W_{i, j}(s, q)-\lambda_{j} \alpha_{j} \sigma^{2}(q) s^{\alpha_{j}} G_{i, j}(s, q) \\
& +\left(\beta(q)-\frac{\lambda_{2} q}{\alpha_{2}-1}-\frac{\lambda_{3} q}{\alpha_{3}-1}\right) \partial_{q} W_{i, j}(s, q)-\left(\lambda_{j}+\eta\right) \sigma^{2}(q) W_{i, j}(s, q)=0,
\end{aligned}
$$


for $s>a$ or $s<b$ and $g<q<h$, where we set

$$
G_{i, 1}(s, q)=-\int_{s}^{\infty} W_{i, 1}(y, q) y^{-\alpha_{1}-1} d y, \quad G_{i, 2}(s, q)=-\int_{s}^{\infty} W_{i, 2}\left(y, \frac{q}{s} y\right) y^{-\alpha_{2}-1} d y
$$

for all $s \geq a$ or $s \leq b$ and $g \leq q \leq h$, and every $i=1,2$, respectively. It follows from the expression in (3.39) and (2.25)-(2.26) that the functions $G_{i, 1}(s, q), i=1,2$, in (3.40) solve the partial differential equations

$$
\begin{aligned}
& \left(\delta-\frac{\lambda_{1}}{\alpha_{1}-1}\right) \sigma^{2}(q) s^{2} \partial_{s s} G_{i, 1}(s, q)+\left(\beta(q)-\frac{\lambda_{1} q}{\alpha_{1}-1}\right) s \partial_{s q} G_{i, 1}(s, q) \\
& +\left(\left(\alpha_{1}+1\right)\left(\delta-\frac{\lambda_{1}}{\alpha_{1}-1}\right)-\left(\lambda_{1}+\eta\right)\right) \sigma^{2}(q) s \partial_{s} G_{i, 1}(s, q)-\lambda_{1} \alpha_{1} \sigma^{2}(q) G_{i, 1}(s, q)=0
\end{aligned}
$$

while the functions $G_{i, 2}(s, q), i=1,2$, in (3.40) solve the partial integro-differential equations

$$
\begin{aligned}
& \left(\delta-\frac{\lambda_{2}}{\alpha_{2}-1}\right) \sigma^{2}(q) s^{2} \partial_{s s} G_{i, 2}(s, q)-\lambda_{2} \alpha_{2} \sigma^{2}(q) G_{i, 2}(s, q) \\
& +\left(\left(\alpha_{2}+1\right)\left(\delta-\frac{\lambda_{2}}{\alpha_{2}-1}\right)-\left(\lambda_{2}+\eta\right)\right) \sigma^{2}(q) s \partial_{s} G_{i, 2}(s, q) \\
& +\left(\beta(q)-\frac{\lambda_{2} q}{\alpha_{2}-1}-\frac{\lambda_{3} q}{\alpha_{3}-1}+\left(\delta-\frac{\lambda_{2}}{\alpha_{2}-1}\right) \sigma^{2}(q) q s^{-\alpha_{2}}\right) \partial_{q} W_{i, 1}(s, q) \\
& -\left(\left(\alpha_{2}-1\right)\left(\delta-\frac{\lambda_{2}}{\alpha_{2}-1}\right)-\left(\lambda_{2}+\eta\right)\right) \sigma^{2}(q) \int_{s}^{b} \partial_{q} W_{i, 2}\left(y, \frac{q}{s} y\right) \frac{q}{s} y^{-\alpha_{2}} d y \\
& +\left(\delta-\frac{\lambda_{2}}{\alpha_{2}-1}\right) \sigma^{2}(q) \int_{s}^{b} \partial_{q q} W_{i, 2}\left(y, \frac{q}{s} y\right)\left(\frac{q}{s}\right)^{2} y^{-\alpha_{2}+1} d y=0
\end{aligned}
$$

for $s>a$ or $s<b$, with the boundary conditions

$$
G_{1, j}(\infty, q)=0, \quad G_{2, j}(b, q)=-\frac{b^{-\alpha_{j}}}{\alpha_{j}}, \quad \partial_{s} G_{1, j}(a+, q)=a^{-\alpha_{j}-1}, \quad \partial_{s} G_{2, j}(b-, q)=b^{-\alpha_{j}-1},
$$

for $g \leq q \leq h$. Note that the third and fourth conditions of (3.43) hold, whenever inequalities $\delta-\lambda_{j} /\left(\alpha_{j}-1\right)<0$ or $\delta-\lambda_{j} /\left(\alpha_{j}-1\right)>0$ are satisfied, respectively, and we have

$$
\partial_{s} H_{i, 2}(s, q)=\int_{s}^{b} \partial_{q} W_{i, 2}\left(y, \frac{q}{s} y\right) \frac{q}{s^{2}} y^{-\alpha_{2}} d y+s^{-\alpha_{2}-1} W_{i, 2}(s, q),
$$

for all $s>a$ and $s<b$ and every $i=1,2$.

Let us first consider the case of $\delta-\lambda_{j} /\left(\alpha_{j}-1\right)<0$. Then, we search for solutions of the equations in (3.41)-(3.42) in the form

$$
G_{i, j}(s, q) \equiv G_{i, j}(s)=D_{i, j, 1} s^{\theta_{j, 1}}+D_{i, j, 2} s^{\theta_{j, 2}},
$$

where $D_{i, j, k}, i, j, k=1,2$, are some arbitrary constants and $\theta_{j, k}, j, k=1,2$, are given by

$$
\theta_{j, k}=\frac{\left(\lambda_{j}+\eta\right)\left(\alpha_{j}-1\right)}{2\left(\delta\left(\alpha_{j}-1\right)-\lambda_{j}\right)}-\frac{\alpha_{j}}{2}+(-1)^{k} \sqrt{\left(\frac{\left(\lambda_{j}+\eta\right)\left(\alpha_{j}-1\right)}{2\left(\delta\left(\alpha_{j}-1\right)-\lambda_{j}\right)}-\frac{\alpha_{j}}{2}\right)^{2}+\frac{\lambda_{j} \alpha_{j}\left(\alpha_{j}-1\right)}{\delta\left(\alpha_{j}-1\right)-\lambda_{j}}}
$$


so that $\theta_{j, 1}<-\alpha_{j}<1-\alpha_{j}<\theta_{j, 2}$ holds. Hence, we obtain from (3.40) that the candidate solution for the system in (2.24)-(2.26) admits the representation

$$
W_{i, j}(s, q) \equiv W_{i, j}(s)=D_{i, j, 1} \theta_{j, 1} s^{\alpha_{j}+\theta_{j, 1}}+D_{i, j, 2} \theta_{j, 2} s^{\alpha_{j}+\theta_{j, 2}},
$$

where we have $\alpha_{j}+\theta_{j, 1}<0<1<\alpha_{j}+\theta_{j, 2}, j=1,2$. Thus, we see that $D_{1, j, 2}=D_{2, j, 1}=0$ should hold in (3.45) and (3.47), since otherwise $W_{i, j}(s) \rightarrow \pm \infty, i, j=1,2$, as $s \uparrow \infty$ and $s \downarrow 0$, respectively, that must be excluded, by virtue of the fact that the functions $W_{i}^{*}(s)$, $i=1,2$, in (2.13) are bounded. Then, by applying the third and second boundary conditions of (3.43) to the function in (3.45), we obtain that the equalities

$$
D_{1, j, 1} \theta_{j, 1} a^{\theta_{j, 1}-1}=a^{-\alpha_{j}-1} \quad \text { and } \quad D_{2, j, 2} b^{\theta_{j, 2}}=-b^{-\alpha_{j}} / \alpha_{j}
$$

are satisfied, for every $j=1,2$. Hence, solving the equations in (3.48), we conclude that the candidate solutions $W_{1, j}(s, q ; a)=W_{1, j}(s ; a)$ and $W_{2, j}(s, q ; b)=W_{2, j}(s ; b), j=1,2$, have the form

$$
W_{1, j}(s ; a)=(s / a)^{\alpha_{j}+\theta_{j, 1}} \quad \text { and } \quad W_{2, j}(s ; b)=-\left(\theta_{j, 2} / \alpha_{j}\right)(s / b)^{\alpha_{j}+\theta_{j, 2}}
$$

for all $s>a$ and $s<b$, respectively.

Let us now consider the case of $\delta-\lambda_{j} /\left(\alpha_{j}-1\right)>0$. In this case, we observe that the equalities $D_{1, j, k}=0, k=1,2$, should hold in (3.45) and (3.47), for $j=1,2$, since otherwise $W_{1, j}(s) \rightarrow \pm \infty$ as $s \uparrow \infty$, that must be excluded by virtue of the fact that the functions $W_{i}^{*}(s)$, $i=1,2$, in (2.13) are bounded. This particularly yields that $W_{1, j}(s, q ; a)=W_{1, j}(s ; a)=0$, for all $s>a$ and every $j=1,2$, that corresponds to the fact that the increasing process $S$ never reaches the point $a$ when started at some $s>a$. Moreover, by applying the second and fourth conditions of (3.43) to the function in (3.45), we obtain that the equalities

$$
D_{2, j, 1} b^{\theta_{j, 1}}+D_{2, j, 2} b^{\theta_{j, 2}}=-b^{-\alpha_{j}} / \alpha_{j} \quad \text { and } \quad D_{2, j, 1} \theta_{j, 1} b^{\theta_{j, 1}-1}+D_{2, j, 2} \theta_{j, 2} b^{\theta_{j, 2}-1}=b^{-\alpha_{j}-1}
$$

are satisfied, for every $j=2,3$. Hence, solving the equations in (3.50), we conclude that the candidate solutions $W_{1, j}(s, q ; a)=W_{1, j}(s ; a)$ and $W_{2, j}(s, q ; b)=W_{2, j}(s ; b), j=1,2$, has the form

$$
W_{1, j}(s ; a)=0 \quad \text { and } \quad W_{2, j}(s ; b)=\frac{\theta_{j, 1}\left(\alpha_{j}-\theta_{j, 2}\right)}{\alpha_{j}\left(\theta_{j, 1}-\theta_{j, 2}\right)}\left(\frac{s}{b}\right)^{\alpha_{j}+\theta_{j, 1}}+\frac{\theta_{j, 2}\left(\alpha_{j}-\theta_{j, 1}\right)}{\alpha_{j}\left(\theta_{j, 2}-\theta_{j, 1}\right)}\left(\frac{s}{b}\right)^{\alpha_{j}+\theta_{j, 2}}
$$

for all $s>a$ and $s<b$, and every $j=1,2$.

Let us finally consider the case of $\delta-\lambda_{j} /\left(\alpha_{j}-1\right)=0$. Then, we search for solutions of the equations in (3.41)-(3.42) in the form

$$
G_{i, j}(s, q) \equiv G_{i, j}(s)=D_{i, j, 0} s^{\theta_{j, 0}}
$$

where $D_{i, j, 0}, i, j=1,2$, are some arbitrary constants and $\theta_{j, 0}=-\lambda_{j} \alpha_{j} /\left(\lambda_{j}+\eta\right)$. Hence, we obtain from (3.40) that the candidate solution for the system in (2.27)-(2.29) admits the representation

$$
W_{i, j}(s, q) \equiv W_{i, j}(s)=D_{i, j, 0} \theta_{j, 0} s^{\alpha_{j}+\theta_{j, 0}}
$$


for $s<b$ and every $j=1,2$. Here, the constants $D_{i, j, 0}, i, j=1,2$, are uniquely determined by the second equation in (3.43). Hence, solving the equation

$$
D_{2, j, 0} b^{\theta_{j, 0}}=-b^{-\alpha_{j}} / \alpha_{j}
$$

we conclude that the candidate solutions $W_{1, j}(s, q ; a)=W_{1, j}(s ; a)$ and $W_{2, j}(s, q ; b)=W_{2, j}(s ; b)$, $j=1,2$, have the form

$$
W_{1, j}(s ; a)=0 \quad \text { and } \quad W_{2, j}(s ; b)=-\left(\theta_{j, 0} / \alpha_{j}\right)(s / b)^{\alpha_{j}+\theta_{j, 0}}
$$

for all $s>a$ and $s<b$, and every $j=1,2$.

\section{Main result and proof}

In this section, taking into account the facts proved above, we formulate and prove the main results of the paper. We present the solution to the two-dimensional stopping problems of (2.11)-(2.12) in the case of $\lambda_{j}>0$, for only one of $j=1,2,3$. The proof of this assertion is based on an extension of the arguments from [21; Theorem 4.1] to the two-dimensional jump case. Another related verification assertion was proved in [18; Theorem 4.1] for a model of a two-dimensional continuous diffusion-type process with switching coefficients.

Theorem 4.1 Suppose that $\alpha_{2}>\theta_{i}+1$ holds, for certain $\theta_{i}, i=1,2$, which are specified below. Assume that the coefficients $\sigma(q)>0$ and $\beta(q) \in \mathbb{R}$ of the jump-diffusion process $(S, Q)$ defined by (2.1)-(2.2) are continuously differentiable functions of at most linear growth, for all $q>0$, and the condition of (2.7) holds, for all $t \geq 0$. Then, the functionals $V_{1}^{*}(s, q)=V_{1}^{*}(s, q ; a ; g, h)$ and $V_{2}^{*}(s, q)=V_{2}^{*}(s, q ; b ; g, h)$ from (2.11)-(2.12) of the associated with $(S, Q)$ random times $\tau_{a}^{-}, \tau_{b}^{+}$and $\zeta_{g}^{-}, \zeta_{h}^{+}$from (2.5)-(2.6) admit the representations

$$
V_{1}^{*}(s, q ; b ; g, h)=U_{1}(q ; g, h) W_{1}(s ; a) \quad \text { and } \quad V_{2}^{*}(s, q ; b ; g, h)=U_{2}(q ; g, h) W_{2}(s ; b)
$$

for all $s>a$ or $s<b$ and $g<q<h$, and any $0<a<b<\infty$ and $0 \leq g<h<\infty$ fixed, where the functions $U_{i}(q ; g, h), i=1,2$, as well as $W_{1}(s ; a)$ and $W_{2}(s ; b)$ are specified as follows:

(i) If $\lambda_{j}>0$ and $\lambda_{5-j}=0$, for some $j=2,3$, either the inequality $\beta_{i}(q)-\xi_{i} q<0$ or $\beta_{i}(q)-\xi_{i} q>0$ holds, for all $g \leq q \leq h$, with $\xi_{i}$ from (2.16), and $\theta_{i}, i=1,2$, take the form of (3.46), then the functions $U_{i}(q ; g, h), i=1,2$, are given by either (3.12) or (3.13), respectively.

(ii) If $\lambda_{j}>0$ and $\lambda_{5-j}=0$, for some $j=2,3$, the condition of (2.30) or (2.31) holds, and $\theta_{i}, i=1,2$, take the form of (3.46), then the functions $U_{i}(q ; g, h), i=1,2$, coincide with $U_{i, j}(q ; h), i=1,2$, from (3.13) or $U_{i, j}^{+}(q ; h), i=1,2$, from (3.19), for all $f<q<h$, and with $U_{i, j}^{-}(q ; g, f)$ from (3.17)-(3.18) or $U_{i, j}^{-}(q ; f, h)$ from (3.19), for all $g<q<f$, respectively.

(iii) If $\lambda_{j}>0$ and $\lambda_{3-j}=0$, for some $j=1,2$, then the functions $W_{1}(s ; a)$ and $W_{2}(s ; b)$ are given by either (3.49) or (3.51) with $\theta_{j, k}, k=1,2$, from (3.46), whenever either $\delta-\lambda_{j} /\left(\alpha_{j}-\right.$ $1)<0$ or $\delta-\lambda_{j} /\left(\alpha_{j}-1\right)<0$ holds, respectively, and by (3.55) with $\theta_{j, 0}=-\lambda_{j} \alpha_{j} /\left(\lambda_{j}+\eta\right)$, whenever $\delta-\lambda_{j} /\left(\alpha_{j}-1\right)=0$ holds. 
Since all the parts of the assertions formulated above are proved using similar arguments, we only give a proof for the two-dimensional stopping problem related to the value function $V_{1}^{*}(s, q ; a ; g, h)$ in $(2.11)$.

Proof In order to verify the assertion stated above, it remains to show that the function on the right-hand side of (4.1) coincides with the value function in (2.11). For this purpose, let us denote by $V_{1}(s, q)$ the right-hand side of the first expression in (4.1). It follows from the standard arguments of the previous section that the function $V_{1}(s, q)$ solves the partial integrodifferential boundary-value problem of (2.19)-(2.23) with (2.17). Then, taking into account the fact that the function $V_{1}(s, q)$ is bounded on $(0, \infty)^{2}$, we can construct a sequence of twice continuously differentiable bounded functions $\left(V_{1, k}\right)_{k \in \mathbb{N}}$ on $(0, \infty)^{2}$ such that $\mid V_{1, k}(s, q)$ $V_{1}(s, q) \mid \leq 1$ holds, for all $(s, q) \in(0, \infty)^{2}$, as well as $V_{1, k}(s, q)=V_{1}(s, q)$ holds, for $(s, q) \in$ $(0, \infty)^{2}$, apart from $(s, q) \in((a-1 / k, a) \times(g-1 / k, h+1 / k))$ and $(s, q) \in[a, \infty) \times((g-$ $1 / k, g) \cup[h, h+1 / k))$. Note that, by virtue of the structure of the functions constructed above, we clearly have $V_{1, k}(s, q) \rightarrow V_{1}(s, q)$ as $k \rightarrow \infty$, for all $(s, q) \in(0, \infty)^{2}$. By applying the Itô's formula for semimartingales to the process $e^{-(\eta+\varkappa) A_{t}} V_{1, k}\left(S_{t}, Q_{t}\right)$, we obtain that the expression

$$
\begin{aligned}
& e^{-(\eta+\varkappa) A_{\tau_{a}^{-} \wedge \zeta_{g}^{-} \wedge \zeta_{h}^{+} \wedge t}} V_{1, k}\left(S_{\tau_{a}^{-} \wedge \zeta_{g}^{-} \wedge \zeta_{h}^{+} \wedge t}, Q_{\tau_{a}^{-} \wedge \zeta_{g}^{-} \wedge \zeta_{h}^{+} \wedge t}\right)=V_{1, k}(s, q) \\
& +\int_{0}^{\tau_{a}^{-} \wedge \zeta_{g}^{-} \wedge \zeta_{h}^{+} \wedge t} e^{-(\eta+\varkappa) A_{u}}\left(\mathbb{L}_{(S, Q)} V_{1, k}-(\eta+\varkappa) \sigma^{2}\left(Q_{u}\right) V_{1, k}\right)\left(S_{u}, Q_{u}\right) d u+M_{\tau_{a}^{-} \wedge \zeta_{g}^{-} \wedge \zeta_{h}^{+} \wedge t}^{k}
\end{aligned}
$$

holds, for all $t \geq 0$, and the stopping times $\tau_{a}^{-}$and $\zeta_{g}^{-}, \zeta_{h}^{+}$from (2.5)-(2.6). Here, the process $M^{k}=\left(M_{t}^{k}\right)_{t \geq 0}$ defined by

$$
\begin{aligned}
M_{t}^{k}= & \int_{0}^{t} \int e^{-(\eta+\varkappa) A_{u}}\left(V_{1, k}\left(S_{u-} e^{x}, Q_{u-}\right)-V_{1, k}\left(S_{u-}, Q_{u-}\right)\right)\left(\mu_{1}-\nu_{1}\right)(d u, d x) \\
& +\int_{0}^{t} \int e^{-(\eta+\varkappa) A_{u}}\left(V_{1, k}\left(S_{u-} e^{x}, Q_{u-} e^{x}\right)-V_{1, k}\left(S_{u-}, Q_{u-}\right)\right)\left(\mu_{2}-\nu_{2}\right)(d u, d x) \\
& +\int_{0}^{t} \int e^{-(\eta+\varkappa) A_{u}}\left(V_{1, k}\left(S_{u-}, Q_{u-} e^{x}\right)-V_{1, k}\left(S_{u-}, Q_{u-}\right)\right)\left(\mu_{3}-\nu_{3}\right)(d u, d x)
\end{aligned}
$$

is a local martingale under $P_{s, q}$. Note that, since $\sigma^{2}(q)$ is a bounded function on $g<q<h$, it follows from the inequality $\left|V_{1, k}(s, q)-V_{1}(s, q)\right| \leq 1$ that we have

$$
\begin{aligned}
& \left|\left(\mathbb{L}_{(S, Q)} V_{1, k}-(\eta+\varkappa) \sigma^{2}(q) V_{1, k}\right)(s, q)\right| \\
& \leq \lambda_{2} \alpha_{2} \sigma^{2}(q) \int_{\log h-\log q}^{\log (h+1 / k)-\log q}\left|V_{1, k}\left(s e^{x}, q e^{x}\right)-V_{1}\left(s e^{x}, q e^{x}\right)\right| d x \\
& \quad+\lambda_{3} \alpha_{3} \int_{\log h-\log q}^{\log (h+1 / k)-\log q}\left|V_{1, k}\left(s, q e^{x}\right)-V_{1}\left(s, q e^{x}\right)\right| d x \\
& \leq \lambda_{2} \alpha_{2} \sigma^{2}(q) \log \left(\frac{h+1 / k}{h}\right)+\lambda_{3} \alpha_{3} \log \left(\frac{h+1 / k}{h}\right) \rightarrow 0
\end{aligned}
$$

as $k \rightarrow \infty$, uniformly on $(s, q) \in(a, \infty) \times(g, h)$. Hence, we obtain from the expression in (4.2) and the fact that $V_{1, k}(s, q)$ is bounded that the inequality

$$
\left|M_{t}^{k}\right| \leq C+\left(\lambda_{2} \alpha_{2} \sigma^{2}(q) \log \left(\frac{h+1 / k}{h}\right)+\lambda_{3} \alpha_{3} \log \left(\frac{h+1 / k}{h}\right)\right) t
$$


holds, for some constant $C>0$ and all $t \geq 0$, so that the process $M^{k}$ is a (bounded) martingale. Thus, taking the expectation with respect to $P_{s, q}$ in (4.2), by means of the Doob's optional sampling theorem (see, e.g. [27; Chapter I, Theorem 1.39]), we get

$$
\begin{aligned}
E_{s, q}\left[e^{-(\eta+\varkappa) A_{\tau_{a}^{-} \wedge \zeta_{g}^{-} \wedge \zeta_{h}^{+} \wedge t}} V_{1, k}\left(S_{\tau_{a}^{-} \wedge \zeta_{g}^{-} \wedge \zeta_{h}^{+} \wedge t}, Q_{\tau_{a}^{-} \wedge \zeta_{g}^{-} \wedge \zeta_{h}^{+} \wedge t}\right)\right. \\
\left.\quad-\int_{0}^{\tau_{a}^{-} \wedge \zeta_{g}^{-} \wedge \zeta_{h}^{+} \wedge t} e^{-(\eta+\varkappa) A_{u}}\left(\mathbb{L}_{(S, Q)} V_{1, k}-(\eta+\varkappa) \sigma^{2}\left(Q_{u}\right) V_{1, k}\right)\left(S_{u}, Q_{u}\right) d u\right]=V_{1, k}(s, q),
\end{aligned}
$$

for all $t \geq 0$ and $(s, q) \in(a, \infty) \times(g, h)$. Note that, by virtue of the fact that $V_{1, k}(s, q) \rightarrow$ $V_{1}(s, q)$ as $k \rightarrow \infty$, for all $(s, q) \in(0, \infty)^{2}$, except $q=h$ in case (i), as well as $P_{s, q}\left(Q_{\zeta_{h}^{+}}=h\right)=0$ holds, we see that $V_{1, k}\left(S_{\tau_{a}^{-} \wedge \zeta_{g}^{-} \wedge \zeta_{h}^{+} \wedge t}, Q_{\tau_{a}^{-} \wedge \zeta_{g}^{-} \wedge \zeta_{h}^{+} \wedge t}\right) \rightarrow V_{1}\left(S_{\tau_{a}^{-} \wedge \zeta_{g}^{-} \wedge \zeta_{h}^{+} \wedge t}, Q_{\tau_{a}^{-} \wedge \zeta_{g}^{-} \wedge \zeta_{h}^{+} \wedge t}\right)$ as $k \rightarrow \infty$ $\left(P_{s, q}\right.$-a.s. $)$, for any $t \geq 0$ and $s, q>0$ fixed. Therefore, by means of the bounded convergence theorem, we obtain

$$
\begin{aligned}
& \lim _{k \rightarrow \infty} E_{s, q}\left[e^{-(\eta+\varkappa) A_{\tau_{a}^{-} \wedge \zeta_{g}^{-} \wedge \zeta_{h}^{+} \wedge t}} V_{1, k}\left(S_{\tau_{a}^{-} \wedge \zeta_{g}^{-} \wedge \zeta_{h}^{+} \wedge t}, Q_{\tau_{a}^{-} \wedge \zeta_{g}^{-} \wedge \zeta_{h}^{+} \wedge t}\right)\right] \\
& =E_{s, q}\left[e^{-(\eta+\varkappa) A_{\tau_{a}^{-} \wedge \zeta_{g}^{-} \wedge \zeta_{h}^{+} \wedge t}} V_{1}\left(S_{\tau_{a}^{-} \wedge \zeta_{g}^{-} \wedge \zeta_{h}^{+} \wedge t}, Q_{\tau_{a}^{-} \wedge \zeta_{g}^{-} \wedge \zeta_{h}^{+} \wedge t}\right)\right]
\end{aligned}
$$

and by uniform convergence of the functions in (4.4), we get

$$
\lim _{k \rightarrow \infty} E_{s, q} \int_{0}^{\tau_{a}^{-} \wedge \zeta_{g}^{-} \wedge \zeta_{h}^{+} \wedge t} e^{-(\eta+\varkappa) A_{u}}\left(\mathbb{L}_{(S, Q)} V_{1, k}-(\eta+\varkappa) \sigma^{2}\left(Q_{u}\right) V_{1, k}\right)\left(S_{u}, Q_{u}\right) d u=0,
$$

for all $t \geq 0$ and $(s, q) \in(a, \infty) \times(g, h)$. Hence, we conclude from the expressions in (4.6)-(4.8) that

$$
E_{s, q}\left[e^{-(\eta+\varkappa) A_{\tau_{a}^{-} \wedge \zeta_{g}^{-} \wedge \zeta_{h}^{+} \wedge t}} V_{1}\left(S_{\tau_{a}^{-} \wedge \zeta_{g}^{-} \wedge \zeta_{h}^{+} \wedge t}, Q_{\tau_{a}^{-} \wedge \zeta_{g}^{-} \wedge \zeta_{h}^{+} \wedge t}\right)\right]=\lim _{k \rightarrow \infty} V_{1, k}(s, q)=V_{1}(s, q)
$$

holds, for all $t \geq 0$ and $(s, q) \in(a, \infty) \times(g, h)$. Therefore, letting $t$ go to infinity and using the boundary conditions in (2.20)-(2.23) as well as the fact that $e^{-(\eta+\varkappa) A_{\tau_{a}^{-} \wedge \zeta_{g}^{-} \wedge \zeta_{h}^{+}}} V_{1}\left(S_{\tau_{a}^{-} \wedge \zeta_{g}^{-} \wedge \zeta_{h}^{+}}, Q_{\tau_{a}^{-} \wedge \zeta_{g}^{-} \wedge \zeta_{h}^{+}}\right)=$ 0 on $\left\{\tau_{a}^{-} \wedge \zeta_{g}^{-} \wedge \zeta_{h}^{+}=\infty\right\}\left(P_{s, q}\right.$-a.s. $)$, we can apply the Lebesgue dominated convergence theorem to the expression in (4.9) and obtain the equalities

$$
\begin{aligned}
& E_{s, q}\left[e^{-(\eta+\varkappa) A_{\tau_{a}^{-}} U\left(Q_{\tau_{a}^{-}}\right.} ; g, h\right) I\left(\tau_{a}^{-}<\zeta_{g}^{-} \wedge \zeta_{h}^{+}\right) \\
& \left.\quad+e^{-(\eta+\varkappa) A_{\zeta_{g}^{-} \wedge \zeta_{h}^{+}}} W_{1}\left(S_{\zeta_{g}^{-} \wedge \zeta_{h}^{+}}, Q_{\zeta_{g}^{-} \wedge \zeta_{h}^{+}} ; a\right) I\left(\zeta_{g}^{-} \wedge \zeta_{h}^{+} \leq \tau_{a}^{-}\right)\right] \\
& =E_{s, q}\left[e^{-(\eta+\varkappa) A_{\tau_{a}^{-} \wedge \zeta_{g}^{-} \wedge \zeta_{h}^{+}}} V_{1}\left(S_{\tau_{a}^{-} \wedge \zeta_{g}^{-} \wedge \zeta_{h}^{+}}, Q_{\tau_{a}^{-} \wedge \zeta_{g}^{-} \wedge \zeta_{h}^{+}}\right)\right]=V_{1}(s, q)
\end{aligned}
$$

for all $s>a$ and $g<q<h$, which directly implies the desired assertion.

Acknowledgments. The authors thank the Editors and the anonymous referee for their valuable suggestions which helped to improve the presentation of the paper. This research was supported by a Small Grant from the Suntory and Toyota International Centres for Economics and Related Disciplines (STICERD) at the London School of Economics and Political Science. 


\section{References}

[1] M. Abramovitz and I. A. Stegun. Handbook of Mathematical Functions with Formulas, Graphs, and Mathematical Tables. National Bureau of Standards. Wiley, New York, 1972.

[2] L. Alili and A. E. Kyprianou. Some remarks on first passage of Lévy processes, the American put and pasting principles. Annals of Applied Probability, 15(3):2062-2080, 2005.

[3] S. Asmussen, F. Avram, and M. R. Pistorius. Russian and American put options under exponential phase-type Lévy models. Stochastic Processes and their Applications, 109(1):79-111, 2004.

[4] S. Assing, S. Jacka, and A. Ocejo. Monotonicity of the value function for a two-dimensional optimal stopping problem. Annals of Applied Probability, 24(4):1554-1584, 082014.

[5] E. Bayraktar, S. Dayanik, and I. Karatzas. The standard Poisson disorder problem revisited. Stochastic Processes and their Applications, 115(9):1437-1450, 2005.

[6] E. Bayraktar and H. V. Poor. Quickest detection of a minimum of two Poisson disorder times. SIAM Journal on Control and Optimization, 46(1):308-331, 2007.

[7] L. Bo, Y. Wang, and X. Yang. First passage times of (reflected) Ornstein-Uhlenbeck processes over random jump boundaries. Journal of Applied Probability, 48(3):723-732, 2011.

[8] M. Davis. A note on the Poisson disorder problem. Banach Center Publications, 1(1):65-72, 1976.

[9] S. Dayanik, H. V. Poor, and S. O. Sezer. Multisource Bayesian sequential change detection. Annals of Applied Probability, 18(2):552-590, 2008.

[10] S. Dayanik and S. O. Sezer. Compound Poisson disorder problem. Mathematics of Operations Research, 31(4):649-672, 2006.

[11] S. Dayanik and S. O. Sezer. Sequential testing of simple hypotheses about compound Poisson processes. Stochastic Processes and their Applications, 116(12):1892-1919, 2006.

[12] E. B. Dynkin. Markov Processes. Springer, Berlin, 1965.

[13] L. Fernández, P. Hieber, and M. Scherer. Double-barrier first-passage times of jump-diffusion processes. Monte Carlo Methods and their Applications, 19(2):107-141, 2013.

[14] J. P. Fouque, G. Papanicolaou, and K. R. Sircar. Derivatives in Financial Markets with Stochastic Volatility. Cambridge University Press, 2000.

[15] P. V. Gapeev. Solving stochastic differential equations. In Research Report 2002-47 of Centre for Mathematical Physics and Stochastics. Aarhus, 2002.

[16] P. V. Gapeev. The disorder problem for compound Poisson processes with exponential jumps. Annals of Applied Probability, 15:487-499, 2005.

[17] P. V. Gapeev. The integral option in a model with jumps. Statistics and Probability Letters, 76:2623-2631, 2008.

[18] P. V. Gapeev, O. Brockhaus, and M. Dubois. On some functionals of the first passage times in models with switching stochastic volatility. International Journal of Theoretical and Applied Finance, 1(1850001):1-20, 2018.

[19] P. V. Gapeev and A. N. Shiryaev. Bayesian quickest detection problems for some diffusion processes. Advances in Applied Probability, 45(1):164-185, 2013.

[20] P. V. Gapeev and Y. I. Stoev. On the construction of non-affine jump-diffusion models. Stochastic Analysis and Applications, 35(5):900-918, 2017.

[21] P. V. Gapeev and Y. I. Stoev. On the Laplace transforms of the first exit times in one-dimensional non-affine jump-diffusion models. Statistics and Probability Letters, 121:152-162, 2017.

[22] T. C. Gard. Introduction to Stochastic Differential Equations. Dekker, New York, 1988. 
[23] J. Gatheral. The Volatility Surface: A Practitioner's Guide. John Wiley and Sons, 2011.

[24] D. I. Hadjiev. The first passage problem for generalized ornstein-uhlenbeck processes with non-positive jumps. 19:80-90, 1985.

[25] S. Iyengar. Hitting lines with two-dimensional Brownian motion. SIAM Journal on Applied Mathematics, 45(6):983-989, 1985.

[26] İ. İyigünler, M. Çă̆lar, and G. Ünal. Exact solvability of stochastic differential equations driven by finite activity Levy processes. Mathematical and Computational Applications, 17:68-82, 2012.

[27] J. Jacod and A. N. Shiryaev. Limit Theorems for Stochastic Processes. Springer, Berlin, 2003.

[28] P. Johnson and G. Peskir. Quickest detection problems for Bessel processes. Annals of Applied Probability, to appear.

[29] J. Kallsen. A didactic note on affine stochastic volatility models. In From Stochastic Calculus to Mathematical Finance, pages 343-368. Springer Berlin Heidelberg, 2006.

[30] S. G. Kou. A jump diffusion model for option pricing. Management Science, 48:1086-1101, 2002.

[31] S. G. Kou and H. Wang. First passage times of a jump diffusion process. Advances in Applied Probability, 35(2):504-531, 2003.

[32] S. G. Kou and H. Wang. Option pricing under a double exponential jump diffusion model. Management Science, 50:1178-1192, 2004.

[33] R. S. Liptser and A. N. Shiryaev. Statistics of Random Processes I, II. Springer, Berlin, 1977.

[34] A. Mijatović and M. R. Pistorius. On the drawdown of completely asymmetric Lévy processes. Stochastic Processes and their Applications, 22:3812-3836, 2012.

[35] E. Mordecki. Optimal stopping for a diffusion with jumps. Finance and Stochastics, 3:227-236, 1999.

[36] E. Mordecki. Optimal stopping and perpetual options for Lévy processes. Finance and Stochastics, 6(4):473-493, 2002.

[37] B. Øksendal. Stochastic Differential Equations. An Introduction with Applications. Springer, Berlin, 1998.

[38] D. Perry, W. Stadje, and S. Zacks. Boundary crossing for the difference of two ordinary or compound Poisson processes. Annals of Operations Research, 113(1):119-132, 2002.

[39] D. Perry, W. Stadje, and S. Zacks. First-exit times for compound Poisson processes for some types of positive and negative jumps. Stochastic Models, 18(1):139-157, 2002.

[40] D. Perry, W. Stadje, and S. Zacks. The first rendezvous time of Brownian motion and compound Poissontype processes. Journal of Applied Probability, 41(4):1059-1070, 2004.

[41] G. Peskir and A. N. Shiryaev. Sequential testing problems for Poisson processes. Annals of Statistics, 28(3):837-859, 2000.

[42] G. Peskir and A. N. Shiryaev. Solving the Poisson disorder problem. In Advances in Finance and Stochastics. Springer 295-312, 2002.

[43] D. Revuz and M. Yor. Continuous Martingales and Brownian Motion. Springer, Berlin, 1999.

[44] A. Sepp. Analytical pricing of double-barrier options under a double-exponential jump diffusion process: Applications of Laplace transform. International Journal of Theoretical and Applied Finance, 7(2):151-175, 2004.

[45] S. Zacks, D. Perry, D. Bshouty, and S. Bar-lev. Distributions of stopping times for compound poisson processes with positive jumps and linear boundaries. Communications in Statistics. Stochastic Models, 15(1):89-101, 1999.

[46] V.F. Zaitsev and A.D. Polyanin. Handbook of Exact Solutions for Ordinary Differential Equations. Taylor and Francis, 2002. 